\title{
A case control study to compare the levels of homocysteine in normal and complicated pregnancies
}

\author{
Neelamma Patil ${ }^{1, *}$, Shilpa Shirdi ${ }^{2}$, AbhigVa V. ${ }^{3}$ \\ ${ }^{1}$ Associate Professor, ${ }^{2,3}$ PG Student, Dept. of Obstetrics and Gynecology, BLDE University, Shri B.M. Patil Medical College, \\ Vijayapur, Karnataka, India
}

*Corresponding Author:

Email: patilneelgiri@ rediffmail.com

\begin{abstract}
Aim: Hyperhomocysteinemia during pregnancy is known to cause endothelial dysfunction as a result of increased oxidative stress or direct toxic effects. The purpose of this study is to find the correlation between Hyperhomocysteinemia and various placental dysfunctional related pregnancy complications.

Materials and Methods: A prospective case control study of 100 women admitted in antenatal ward or Labour room of department of OBG was conducted at Shri B.M. Patil Medical College Hospital and research center Vijayapur. Study Group consisted of 70 pregnant women with different pregnancy complications like Pre eclampsia, Eclampsia, unexplained Abruption, FGR with oligohydramnios and unexplained oligohydramnios. Control Group was of 30 healthy pregnant women. Fasting Homocysteine level was checked in all participants.

Results: Mean level of Homocysteine was significantly raised in all patients with pregnancy complications (study group-26.4 umol/l) compared to patients without complications (control group $-8.4 \mu \mathrm{mol} / \mathrm{L}$ ) with a $\mathrm{p}$ value of $<0.05$. Significantly more number of patients had Hyperhomocysteinemia in each of pregnancy complication compared to control group $(\mathrm{p}<0.05)$. Among them, Eclampsia group had the highest mean value of $29.40 \mu \mathrm{mol} / \mathrm{L}$ followed by unexplained abruption $(24.60 \mu \mathrm{mol} / \mathrm{L})$, Pre Eclampsia $(22.05 \mu \mathrm{mol} / \mathrm{L})$, FGR with oligohydramnios $(21.90 \mu \mathrm{mol} / \mathrm{L})$ and unexplained oligohydramnios $(20.90 \mu \mathrm{mol} / \mathrm{L})$. Patients with high levels of Homocysteine also had poor pregnancy outcome.

Conclusion: In our study serum Homocysteine levels were significantly elevated in various pregnancy complications compared to control group. Patients with Hyperhomocysteinemia also had poor pregnancy outcome. Hence a strong correlation may exist between serum Homocysteine levels and complications of pregnancy related to placental dysfunction.

Clinical significance: Supplementation of folic acid, vitamin B6 and vitamin B12 up to the completion of placentation or throughout pregnancy may be useful to prevent such complications.
\end{abstract}

Keywords: Homocysteine, Hyperhomocysteinemia, Placental Dysfunction, Pregnancy Complications.

\section{Introduction}

Homocysteine is an intermediate product of Methionine metabolism. As methionine cannot be stored in liver, it is demethylated to Homocysteine for storage purpose. ${ }^{1}$ The total plasma Homocysteine level of healthy individual varies with age, gender, geographical areas and environmental factors. Normal values are between 5-15 $\mu \mathrm{mol} / \mathrm{L}^{2}{ }^{2}$ Hyperhomocysteinemia is defined as fasting plasma value more than $15 \mu \mathrm{mol} / \mathrm{L}$. Hyperhomocysteinemia may be a because of deficiency of folic acid, vitamin B6 and B12 or genetic defect of enzyme required for Homocysteine metabolism. ${ }^{3}$

Hyperhomocysteinemia has been known to cause many vascular diseases. But the exact pathogenesis of Hyperhomocysteinemia induced vascular damage is not clearly known. Several factors such as oxidative stress, endothelial dysfunction, smooth muscle cell proliferation and coagulation abnormalities have been attributed to Hyperhomocysteinemia. ${ }^{2}$ These factors might lead to placental dysfunction leading to various pregnancy complications like recurrent pregnancy loss, pre- Eclampsia, Eclampsia, Abruptio placentae and Fetal growth restriction (FGR) with oligohydramnios. ${ }^{4}$ Placental dysfunction might also lead to oligohydramnios without causing FGR. So, an attempt was made find the co relation between Hyperhomocysteinemia and such placental dysfunction related pregnancy complications through this study.

\section{Material and Methods}

The study was conducted on pregnant women who were admitted in labour the ward at the Department of OBG, Shri B M Patil Medical College Hospital \& Research Centre, Vijayapur. It was done on a total of 100 patients. Among them 70 cases ( study group) were of pregnancy complications like -Pre-eclampsia(20), Eclampsia (10), unexplained Abruption(10), fetal growth restriction(FGR) with oligohydramnios(10), unexplained oligohydramnios(10) and 30 cases were of healthy pregnant women (control group).

Preeclampsia was defined as $\mathrm{BP} \geq 140 / 90 \mathrm{mmhg}$, with proteinuria on more than two occasions. Both mild and severe pre eclampsia were included. Eclampsia was defined as generalised convulsions in pregnant women in the presence of Pre Eclampsia. Unexplained Abruption was considered when Abruption was present in the absence of any obvious cause. FGR was diagnosed both clinically and ultra sonographically. 
Unexplained oligohydramnios was considered when amniotic fluid index of $<8$ by ultrasonography was present in the absence of any hypertension, FGR or postmaturity. Control group consisted of pregnant women with no obstetric complications. Only pregnant women between 15-40yrs and 24-42weeks of gestation age were enrolled for the study. Pregnant women having any other medical diseases like cardiovascular diseases, diabetes mellitus, liver diseases, renal diseases, pre existing hypertension, multiple pregnancy and Hemoglobin $<7 \mathrm{gm} \%$ were excluded from the study.

The study was approved by the institutional ethical committee. Informed consent was taken from the patient for peripheral blood sampling. A fasting blood sample was taken after 6-8hrs of fasting. It was taken on admission or within $24 \mathrm{hrs}$ after delivery depending on the fasting status of patient. $5 \mathrm{ml}$ of blood was drawn from antecubital vein \& stored in refrigerator until tested. Homocysteine level was measured by chemiluminiscence method. Routine investigation were done for all patients and special investigations were done depending on the complications in the cases. Statistical analysis was done using $\mathrm{t}$ test \& $\mathrm{z}$ test. P value of $<0.05$ was considered significant.

\section{Results}

Table 1 shows the baseline characteristics of both the groups. There were more number of patients between 28-34weeks in study group compared to control group. Significantly more numbers of primigravidae were seen in study group as most of complications are in primigravida. Past obstetric history was not considered for data analysis. Other baseline parameters were comparable.

It was observed that mean Homocysteine level (Table 2) was more in study group (26.4mmol) compared to control group $(10.2 \mathrm{mmol})$ with a p value $<0.05$. Also in each pregnancy complication subgroup, the level was significantly more compared to the control group ( $\mathrm{p}$ value <0.05). Among them, Eclampsia group had the highest mean value of 29.40 $\mu \mathrm{mol} / \mathrm{L}$ followed by unexplained Abruption (24.60 $\mu \mathrm{mol} / \mathrm{L})$, Pre Eclampsia $(22.05 \mu \mathrm{mol} / \mathrm{L})$, FGR with oligohydramnios $(21.90 \mu \mathrm{mol} / \mathrm{L})$ and unexplained oligohydramnios $(20.90 \mu \mathrm{mol} / \mathrm{L})$. This could show that higher the value, more severe is the complication. Among 30 patients in control group 24 patients had values $<15 \mu \mathrm{mol} / \mathrm{L}$ whereas 58 out of 70 had raised Homocysteine levels in study group, which was statistically significant (table 3). In eclampsia group, none of them had normal values. In each of the pregnancy complications, significantly more number of patients had higher values of Homocysteine.

All the patients could be followed up to look for the pregnancy outcome. It was found that significantly more number of patients (19/30) had vaginal delivery in control group whereas only 29/70 had vaginal delivery in study group (table 4). Study group also had more of preterm deliveries (25/70) compared to control group (5/20). 9 patients had intrauterine deaths before admission in the study group. Only 3 babies required NICU admission in control group whereas 37 babies required NICU admission in study group. There were no cases of neonatal deaths in control group but study group had 3 neonatal deaths.

Table 1: Baseline characteristics among both groups

\begin{tabular}{|l|c|c|c|}
\hline & Control group & Study group & P value \\
\hline Age & $22.5 \mathrm{yr}$ & $26.5 \mathrm{yr}$ & 0.1622 \\
\hline Gestation age & $38.3 \mathrm{wks}$ & $35.2 \mathrm{wks}$ & 0.2620 \\
\hline $\begin{array}{r}\text { Parity } \\
\text { Primi } \\
\text { Multi }\end{array}$ & 10 & 39 & $0.0402^{*}$ \\
\hline
\end{tabular}

*significant

Table 2: Mean Homocysteine levels in both the groups

*significant

\begin{tabular}{|l|c|c|}
\hline & $\begin{array}{c}\text { Mean Homocysteine } \\
\text { Values }\end{array}$ & P value \\
\hline 1. Control Group & $10.2 \mathrm{umol} / 1$ & \\
\hline 2. Study group & $26.4 \mathrm{umol}$ & $<0.05^{*}$ \\
\hline a. Preeclampsia & $22.05 \mathrm{umol} / 1$ & $<0.05^{*}$ \\
\hline b. Eclampsia & $29.4 \mathrm{umol} / 1$ & $<0.05^{*}$ \\
\hline c. Abruption placenta & $24.60 \mathrm{umol} / 1$ & $<0.05^{*}$ \\
\hline d. FGR with Oligohydramnios & $21.9 \mathrm{umol} /$ & $<0.05^{*}$ \\
\hline e. Unexplained oligohydramnios & $20.9 \mathrm{umol}$ & $<0.05^{*}$ \\
\hline
\end{tabular}


Table 3: Number of patients with normal and abnormal Homocysteine levels

\begin{tabular}{|l|c|c|c|}
\hline & Normal values & $\begin{array}{c}\text { Abnormal } \\
\text { values }\end{array}$ & P value \\
\hline 1.Control group(30) & 24 & 6 & $<0.05^{*}$ \\
\hline 2.Study group(70) & 12 & 58 & $<0.005^{* *}$ \\
\hline a.Preeclampsia(20) & 5 & 15 & $<0.05^{*}$ \\
\hline b.Eclampsia(10) & none & 10 & $<0.05^{*}$ \\
\hline c. Abruption placenta (10) & 1 & 9 & $<0.05^{*}$ \\
\hline $\begin{array}{c}\text { d.FGR with } \\
\text { Oligohydramnios(20) }\end{array}$ & 4 & 16 & $<0.05^{*}$ \\
\hline $\begin{array}{l}\text { e. Unexplained } \\
\text { oligohydramnios(10) }\end{array}$ & 2 & 8 & $<0.05^{*}$ \\
\hline
\end{tabular}

* significant, ${ }^{* *}$ highly significant

Table 4: Pregnancy outcome among both the groups

\begin{tabular}{|l|c|c|c|c|c|}
\hline \multicolumn{1}{|c|}{ Outcome } & $\begin{array}{c}\text { Control } \\
(\mathbf{3 0})\end{array}$ & $\mathbf{\%}$ & $\begin{array}{c}\text { Study } \\
(\mathbf{7 0})\end{array}$ & $\mathbf{\%}$ & P value \\
\hline 1.Vaginal delivery & 19 & 63.33 & 29 & 41.43 & $0.0445^{*}$ \\
\hline $\begin{array}{l}\text { 2.Preterm vaginal } \\
\text { delivery }\end{array}$ & 3 & 10 & 9 & 12.86 & 0.6870 \\
\hline 3.Term LSCS & 11 & 36.67 & 41 & 58.57 & $0.0445^{*}$ \\
\hline 4.Preterm LSCS & 2 & 6.67 & 16 & 22.86 & 0.0535 \\
\hline 5.NICU admission & 3 & 10 & 37 & 52.86 & $<0.0001^{* *}$ \\
\hline
\end{tabular}

*significant, ${ }^{* *}$ highly significant

\section{Discussion}

Homocysteine levels are very important during pregnancy. Hyperhomocysteinemia appears to cause endothelial dysfunction through direct toxic and oxidative stress. It can damage decidual blood vessels and result in faulty placentation with its consequences. ${ }^{3}$

In our study mean Homocysteine levels were significantly high in study group $(26.4 \mathrm{mmol})$ compared to control group $(10.2 \mathrm{mmol})$ with $\mathrm{p}$ value of $<0.05$. Control group mean value was less than the normal cut off value. When individual complication sub group was considered the level was still more than the normal. The difference was also significant when compared to control group $(\mathrm{p}<0.005)$. This shows that Hyperhomocysteinemia is related to pregnancy complications caused by placental dysfunction. Among the pregnancy complications studied Preeclampsia, Eclampsia, FGR and Abruption have already been known to be caused by placental dysfunction due to various vasculopathies. But unexplained oligohydramnios has not been evaluated much for its etiology. Our study shows that it may be due to some kind of placental dysfunction directly or indirectly related to Hyperhomocysteinemia. Till now there are no studies on Hyperhomocysteinemia as an etiology in otherwise unexplained Abruption. But in our study significantly more number of patients had increased Homocysteine levels. Since our sample size is small both for unexplained oligohydramnios and unexplained Abruption, more studies with larger sample size will be required to prove the association.
In a study conducted by Sunita G, it was concluded that the mean level of Homocysteine level was more in women with severe Pre- Eclampsia as compared to those with mild Pre eclampsia and it also showed that there was increased incidence of FGR, IUD, still birth, Abruption and Cesarean section. ${ }^{1}$ But since in our study all the patients in pre Eclampsia group were cases of severe preeclampsia, we could not compare Homocysteine levels between mild and severe preeclampsia.

Yeter A. et al in their study found that patients with FGR had significantly higher level of Homocysteine levels compared to control group. They also found that there was a possible association between Homocysteine levels and Pulsatality index (PI) and Resistance index (RI) and middle cerebral artery Doppler velocimetry. ${ }^{5}$ A study was done by Furness D on 137 patients. Among the patients who had high Homocysteine levels and reduced RBC folate levels developed FGR $(\mathrm{p}<0.001){ }^{6}$ Georgio, Alexis $\mathrm{S}$ et al studied serum Homocysteine level, Folic acid with Vitamin B12 in pregnancy with Pre Eclampsia and concluded that Hyperhomocysteinemia appears to be more common among pre eclamptic women than the normal controls ( $p$ value $<0.001){ }^{2}$ Pre eclamptic patients are at a high risk of abruption. But the cause in unexplained abruption has not been evaluated. In our study, significantly more number of patients had Hyperhomocysteinemia. So, it shows that Hyperhomocysteinemia might lead to abruption without causing preeclampsia. 
A study was done to know the role of first trimester Homocysteine levels and pregnancy outcome by Mariano M. Patients who developed hypertensive disorders in pregnancy, oligohydramnios, meconium stained amniotic fluid, pregnancy loss and had low birth weight babies had significantly high level of Homocysteine. ${ }^{7}$ In another study by Po-jencheng in 2016 on prognostic value of cardiovascular disease risk factors on the severity of pre eclampsia measured in first trimester suggested that, Homocysteine related endothelial dysfunction can be a contributor for abnormal implantation which later leads to early onset preeclampsia and/or preeclampsia with FGR. ${ }^{8}$ Noopur J. conducted a study to know the correlation of serum Homocysteine levels and pregnancy outcome. In this study serum Homocysteine levels and MTHFR gene polymorphism of pregnant women between $14-24$ weeks of gestation were estimated. Pregnant women with complications were found to have high Homocysteine levels when compared to pregnant women without complications though statistically not significant. ${ }^{9}$

Pandy K. et al in their study showed that treating the Hyperhomocysteinemia with Homocysteine lowering agents for 6 weeks reduced the levels significantly ${ }^{4}$. In one more randomized control trial conducted by Manizheh, it was concluded that high dose of folic acid $(5 \mathrm{mg})$ was significantly more effective in reducing Homocysteine level than 500micrograms when started from early pregnancy and continued throughout pregnancy. It was also noted that early abortion rate was less and birth weight was more in patients who took high dose of folic acid. ${ }^{10}$ But larger studies are required to prove whether supplementing these vitamins will really reduce all the complications or not.

Since all the study and control group patients were either in labour or induced due to pregnancy complications, we could study the pregnancy outcome in all patients. Significantly more number of patients required caesarean section in the study group and more number of babies required NICU admission, showing that Hyperhomocysteinemia leads to poor pregnancy outcome due to various pregnancy complications.

\section{Limitations of this study}

Sample size is small for each of the complication in the study group.

\section{Conclusion}

Our study shows that Homocysteine levels are significantly high in various pregnancy complications like Preeclampsia, Eclampsia, unexplained Abruption, FGR and unexplained oligohydramnios compared to control group without any pregnancy complication. Patients with Hyperhomocysteinemia also had poor pregnancy outcome compared to control group. Hence a strong correlation may exist between serum
Homocysteine levels and complications of pregnancy related to placental dysfunction. Supplementation of folic acid, vitamin B6 and vitamin B12 up to the completion of placentation or throughout pregnancy may be useful to prevent such complications. Larger studies are required to show such benefits.

\section{Clinical significance}

Supplementation of folic acid, vitamin B6 and vitamin B12 up to the completion of placentation or throughout pregnancy may be useful to prevent such complications. Larger studies are required to show such benefits.

Acknowledgments: This study was funded by BLDE University, Vijayapur, Karnataka, India.

Conflict of interest: There are no conflicts of interest to be disclosed.

Ethical approval: Institutional ethical committee clearance obtained.

Informed consent: Informed consent was obtained from all the participants.

\section{References}

1. SunitaGhike, Sheela jain, Bhavnakumare, chaitnyashembekar et al. A Study of serum homocysteine levels during normal pregnancy and preeclampsia. Journal of South Asian federation of Obstetrics and Gynaecology. May 2011;3(2);71-4.

2. Arpita P. Patel,Chandan Chakrabarti, Arjun Singh, Jatin D Patel, Hitesh A.Mewada, S.L.Sharma et al. Effect of homocysteine, vitamin B12 and folic acid during pregnancy. NHL journal of medical sciences. July 2012;1(1);27-31.

3. Georgios, AlexisAreti, Anastiosis, Violeta Michalis et al. Homocysteine,folic acid and B12 serum levels in pregnancy complicated with preeclampsia.ArchGynecolObstet 2007;275;121-4.

4. Pandey Kiran, DubayPreeti,TyagiGeetika,Bhagoliwal et al. Hyperhomocsteinemia as a Risk factor for IUGR. The Journal of Obsterics and Gynecology of India. JulyAugust 2012;62(4);406-8.

5. Yeter A, Toppcu HO, Guzel AL, Ozgu E, Danisman N. Maternal plasma homocyseteine levels in intra uterine growth retardation. J Matern Fetal Neonatal Med. April 2015;28(6);709-12.

6. Furness D, Fenech G, Khong T Y, Roberts and Hague. Folate, VitaminB 12, Vitamin B6 and homocysteine: impact on pregnancy outcome. Maternal and Child Nutrition. April 2013;9(2);155-66.

7. Mariano Mascarenhas, Syed Habeebullah, and M. G. Sridhar, "Revisiting the Role of First Trimester Homocysteine as an Index of Maternal and Fetal Outcome," Journal of Pregnancy, vol. 2014, Article ID 123024, 6 pages, 2014. doi:10.1155/2014/123024

8. Cheng P-J, Huang S-Y, Su S-Y, Hsiao C-H, Peng H-H, Duan T. Prognostic Value of Cardiovascular Disease Risk Factors Measured in the First-Trimester on the Severity of Preeclampsia. Elejalde. R, ed. Medicine. 2016;95(5):e2653. doi:10.1097/MD.0000000000002653.

9. Noopur Jaiswal, Anjoo Agarwal, Smriti Agarwal, Vinita Das, Amita Pandey, Rajendra Singh. Correlation of serum Homocysteine Levels and pregnancy outcome: the 
dilemma continues. Int J ReprodContraceptObstet Gynecol. 2016; 5(2);291-395.

10. ManizhehSayyah-Melli, Amir Ghorbanihaghjo, MahastiAlizadeh, MaryamalsdatKazemi-Shishvan, SanamBidadi. The Effect Of High Dose Folic Acid Throughout Pregnancy on Homocysteine (Hcy)

Concentration and Pre-Eclampsia: A Randomized Clinical Trail. PLoS One. 2016;11(5): e0154400. 